\title{
Joubert syndrome (and related disorders) (OMIM 213300)
}

Joubert syndrome (JS) and related disorders are characterized by the 'molar tooth sign' (cerebellar vermis hypoplasia and brainstem anomalies) on MRI, hypotonia, developmental delay, ataxia, irregular breathing pattern and abnormal eye movements. Combinations of additional features such as polydactyly, ocular coloboma, retinal dystrophy, renal disease, hepatic fibrosis, encephalocele, and other brain malformations define clinical sub-types. Recent identification of the NPHP1, AHI1, and CEP290 genes has started to reveal the molecular basis of JS, which may implicate the primary cilium in these disorders. Additional genes remain to be identified.

\section{In brief}

- Joubert syndrome (JS) is a rare autosomal recessive brain malformation disorder with the key finding of cerebellar vermis hypoplasia with a complex brainstem malformation that comprises the molar tooth sign (MTS) on axial MRI images.

- Although the MTS is a necessary feature of JS, other clinical elements that support a diagnosis include hypotonia, developmental delay/mental retardation, cerebellar ataxia, abnormal eye movements, and irregular respiratory pattern.

- Other clinical features define subtypes of JS, termed Joubert syndrome and related disorders (JSRD). These include occipital encephalocele, polymicrogyria, polydactyly, ocular coloboma, retinal dystrophy, cystic kidney disease, nephronophthisis, and hepatic fibrosis.

- Some degree of mental retardation is usually present in JSRD and is typically moderate in severity; affected individuals should receive developmental interventions.

- Homozygous deletion of the NPHP1 gene is causative in $\sim 1-2 \%$ of JSRD subjects with a distinctive form of the
MTS, retinal dystrophy in some cases, and juvenile nephronophthisis, a medullary cystic kidney disease leading to end-stage renal failure.

- Mutations in the AHI1 gene are causative in $~ 10-15 \%$ of JSRD subjects, many of whom exhibit retinal dystrophy, and in some cases, polymicrogyria or later onset nephronophthisis.

- Mutations in the CEP290 (NPHP6) gene are estimated to cause $\sim 10 \%$ of JSRD and are associated with retinal dystrophy and/or congenital blindness, as well as renal disease in some families.

- Two other JS loci have been published (9q34 and pericentromeric 11), but the causative genes have not been identified.

- It is likely that the primary cilium/basal body apparatus is defective in some subtypes of JSRD, but how this causes the complex phenotype remains unknown.

- As some of the medical complications occur with increasing age, based on our current understanding, it is recommended that individuals with JSRD be monitored with regular eye exams for retinal dystrophy and periodic evaluations for renal disease and hepatic fibrosis.
Melissa A Parisi $^{*}{ }^{1}$, Dan Doherty ${ }^{1}$, Phillip F Chance ${ }^{1}$ and lan A Glass ${ }^{1}$

${ }^{1}$ Division of Genetics and Developmental Medicine, Department of Pediatrics, University of Washington School of Medicine, Seattle, WA, USA

European Journal of Human Genetics (2007) 15, 511-521.

doi:10.1038/sj.ejhg.5201648; published online 21 March 2007

Keywords: cerebellar vermis hypoplasia; Joubert syndrome; molar tooth sign; cerebello-oculo-renal syndrome; oculomotor apraxia; nephronophthisis

${ }^{*}$ Correspondence: Dr MA Parisi, Division of Genetics and Developmental Medicine, University of Washington School of Medicine, 1959 N.E. Pacific Street, Box 356320, Seattle, WA 98195-6320, USA.

Tel: + 1206987 2789; Fax: + 1206987 2495;

E-mail: mparisi@u.washington.edu

Received 16 January 2006; revised 27 March 2006; accepted 28 March 2006; published online 21 March 2007

\section{Introduction}

Joubert syndrome (JS) is an autosomal recessive disorder characterized by a specific mid-hindbrain malformation, hypotonia and developmental delay/mental retardation. JS is associated with the 'molar tooth sign' (MTS), a radiologic finding that includes cerebellar vermis hypoplasia and malformation of the brainstem. ${ }^{1}$ An irregular breathing pattern in the newborn period and abnormal eye movements may be observed..$^{2-4}$ Typically, the hypotonia is most prominent during infancy, and cerebellar ataxia develops later. Classic JS is defined as the MTS, hypotonia, developmental delay/mental retardation, \pm oculomotor apraxia (OMA), \pm breathing abnormalities (Figure 1). Some authors include typical facial features in the clinical diagnosis. ${ }^{5}$ Conditions sometimes associated with JS and 


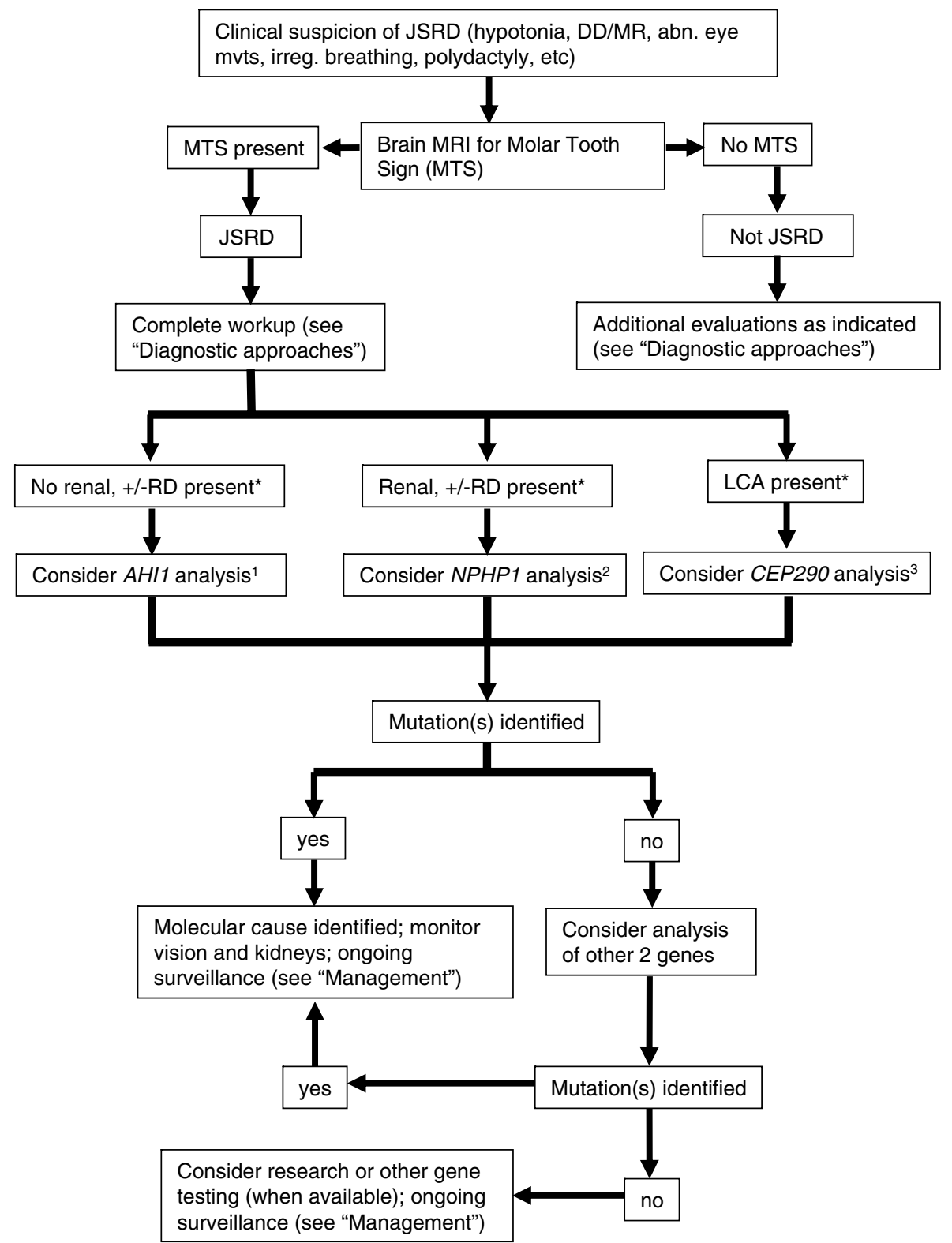

Figure 1 Diagnostic and management algorithm for JSRD. See text for details. This proposed testing strategy is likely to change with the discovery of additional genes and refined genotype-phenotype correlations. *Renal, juvenile nephronophthisis or renal concentrating defect; RD, retinal dystrophy; LCA, Leber congenital amaurosis. ${ }^{1}$ AHI1 mutations are estimated to cause JSRD in $\sim 11 \%$ of subjects; sequencing of all coding exons is available. ${ }^{2}$ Homozygous NPHP1 deletions are estimated to cause JSRD in $\sim 1-2 \%$ of subjects; testing by fluorescence in situ hybridization (FISH) or marker analysis to identify homozygous deletions is available. ${ }^{3}$ CEP290 mutations are estimated to cause JSRD in $\sim 10 \%$ of subjects; sequencing of all coding exons is currently available. JSRD, Joubert syndrome and related disorders, DD/MR, developmental delay/mental retardation, abn. eye mvts., abnormal eye movements (including nystagmus, OMA), irreg. breathing, irregular breathing pattern (tachypnea and/or apnea).

referred to as Joubert syndrome and related disorders (JSRD) include features such as other central nervous system anomalies, polydactyly, ocular coloboma, retinal dystrophy, renal disease including cystic dysplasia or nephronophthisis, and hepatic fibrosis. Some of these findings are not apparent at birth.
Although no direct measurement of the incidence of JS exists, its prevalence in the United States has been estimated as approximately $1: 100000{ }^{6}$ This is likely to be an underestimate due to lack of recognition of the clinical signs or MRI findings and failure to diagnose the condition in more mildly affected individuals. 


\section{Clinical overview}

\section{Core diagnostic criteria}

Although diagnostic criteria for JS have not been established, the clinical features often cited as necessary for the diagnosis of classic JS include: ${ }^{2-6}$

1. Cranial MRI findings demonstrating the MTS on axial imaging with these three components: midline cerebellar vermis hypoplasia, deepened interpeduncular fossa, and thick, elongated superior cerebellar peduncles (see Figure 2; present in $100 \%)^{1,7}$

2. Hypotonia in infancy $(100 \%)$

3. Developmental delay/mental retardation, of variable severity $(100 \%)$

4. One or both of the following (not absolutely required but supportive of the diagnosis):

a. Irregular breathing pattern in infancy (episodic tachypnea and/or apnea)

b. Abnormal eye movements (including nystagmus, jerky eye movements, and OMA or difficulty with smooth visual pursuits).

\section{JSRD and associations}

The disorders that share the MTS have been termed JSRD. ${ }^{8}$ JS may also be considered an oculo-cerebello-renal syndrome because of the coincident involvement of all three organ systems. ${ }^{9}$ Certain clinical features tend to occur in distinctive patterns, such as the combination of retinal and renal involvement, lending support to this proposed nosologic framework; ${ }^{8,10,11}$ however, these associations have not always been consistent. The clinical features and genetic causes of JSRD (where known) are summarized in Table 1 . Whether these conditions represent distinct disorders or share common genetic etiologies with 'classic JS' as defined by the core diagnostic criteria remains to be determined. In some of these conditions, the MTS is always present, whereas in others (such as juvenile nephronophthisis or Senior-L $\varnothing$ ken syndrome), only a subset of individuals with the diagnosis manifests the MTS. ${ }^{11}$ This nosologic classification is inherently limited by the lack of genotype-phenotype correlations and awaits the identification of additional causative genes to provide molecular clarification. The following features by organ system have been reported in individuals with JSRD.

\section{Central nervous system}

Central nervous system involvement may also include fluid collections in the posterior fossa resembling DandyWalker malformation and is observed in $\sim 10 \%$ of subjects. ${ }^{12}$ Although hydrocephalus is uncommon in JS, a few patients develop hydrocephalus requiring shunting even in the absence of a Dandy-Walker malformation. ${ }^{13}$
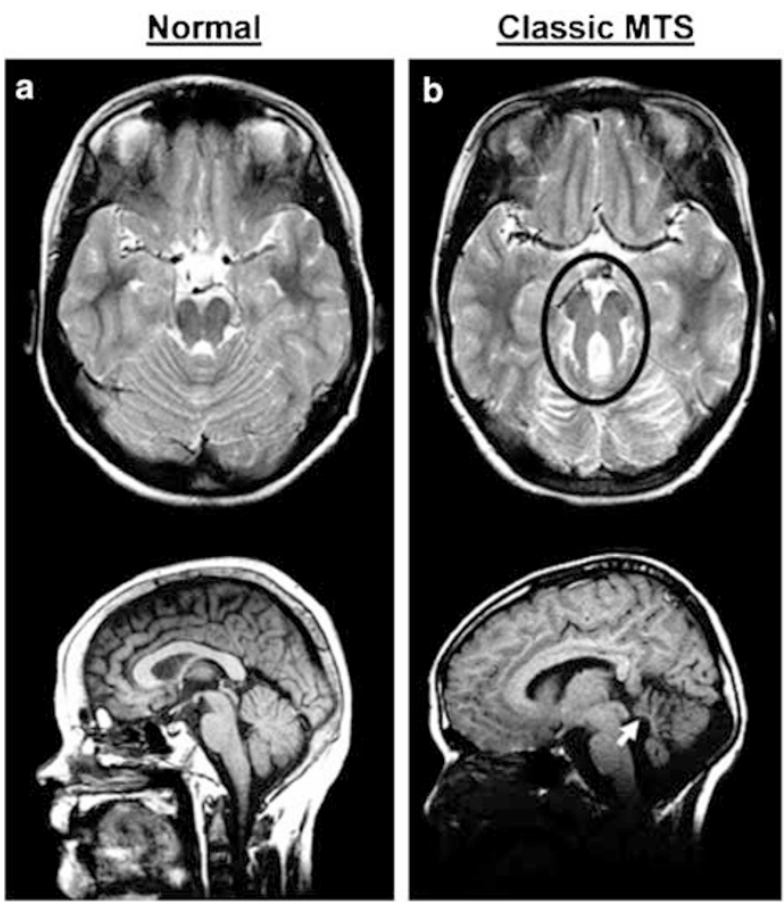

\section{MTS in NPHP1 del}

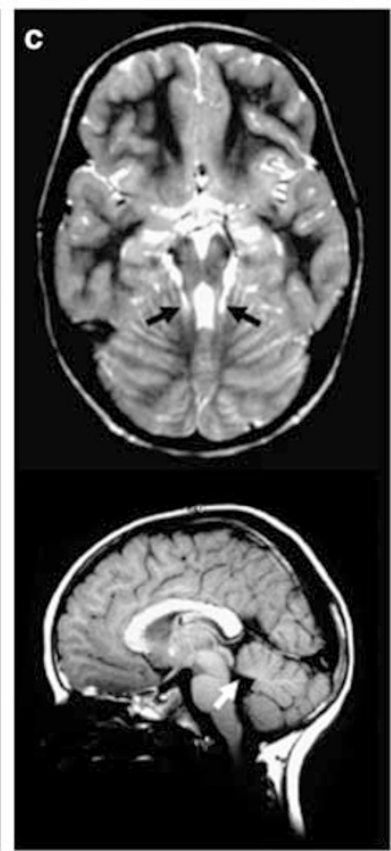

Figure 2 MRI images showing the (a) normal appearance of the cerebellum in comparison with (b) the typical MTS circled in black (top), with elevation of the fourth ventricle and vermis hypoplasia (white arrow) shown on sagittal image (bottom). (c) In the patient with a homozygous NPHP1 deletion, the superior cerebellar peduncles are thin (black arrows, above) and there is less vermis hypoplasia and fourth ventricular elevation (below). 
Table 1 Features in JSRD with the molar tooth sign

\begin{tabular}{|c|c|c|c|c|c|c|c|}
\hline Clinical features & $\begin{array}{l}\text { Joubert } \\
\text { syndrome }\end{array}$ & $\begin{array}{l}\text { Dekaban- } \\
\text { Arima } \\
\text { syndrome }\end{array}$ & $C O A C H^{b}$ & $\begin{array}{c}\text { OFD }{ }^{C} \text { VI } \\
\text { (Varadi-Papp } \\
\text { syndrome) }\end{array}$ & $\begin{array}{l}\text { Joubert-LCA } \\
\text { like }\end{array}$ & $\begin{array}{c}\text { Juvenile } \\
\text { nephronophthisis }\end{array}$ & $S L S$ \\
\hline MTS & + & $(+)$ & $(+)$ & $(+)$ & + & $(+)$ & $(+)$ \\
\hline Hypotonia & + & + & + & + & + & $(+)$ & $(+)$ \\
\hline Mental retardation & + & + & + & + & + & $(+)$ & $(+)$ \\
\hline $\mathrm{OMA}^{\mathrm{e}}$ & $(+)$ & $(+)$ & $(+)$ & $(+)$ & $(+)$ & $(+)$ & $(+)$ \\
\hline Breathing abnormalities ${ }^{f}$ & $(+)$ & $(+)$ & $(+)$ & $(+)$ & $(+)$ & $?$ & $?$ \\
\hline Encephalocele $^{\mathrm{g}}$ & $(+)$ & $(+)$ & $(+)$ & $(+)$ & $(+)$ & - & $(+)$ \\
\hline Polymicrogyria $^{h}$ & $(+)$ & $?$ & $?$ & $?$ & $?$ & $?$ & $?$ \\
\hline Other CNS $S^{1}$ & $(+)$ & ? & ? & $(+)$ & ? & - & - \\
\hline Retinal dystrophy & $(+)^{j}$ & + & $?$ & - & - & - & + \\
\hline $\mathrm{LCA}^{\mathrm{d}}$ & $(+)^{j}$ & + & $(+)$ & $(+)$ & + & $(+)$ & $?$ \\
\hline Coloboma & - & $(+)$ & + & ? & ? & - & - \\
\hline Cystic dysplastic kidneys ${ }^{k}$ & $(+)^{j}$ & + & - & $(+)$ & - & - & - \\
\hline Nephronophthisis ${ }^{k}$ & $(+)^{j}$ & - & $(+)$ & - & - & + & + \\
\hline Hepatic fibrosis & - & $(+)$ & + & - & - & $(+)$ & $(+)$ \\
\hline Polydactyly & $(+)$ & $?$ & Rare & +1 & $(+)$ & - & - \\
\hline Tongue hamartomas/oral frenulae & - & - & - & + & - & - & - \\
\hline Genes & $\begin{array}{c}\text { NPHP1, } A H I 1, \\
\text { CEP290, } \\
\text { others }\end{array}$ & $?$ & $?$ & $?$ & $\begin{array}{c}\text { AHI1, CEP290, } \\
\text { others }\end{array}$ & $\begin{array}{l}\text { NPHP1, 3, 4, } \\
\text { CEP290, others }\end{array}$ & $\begin{array}{l}\text { NPHP1, 3, 4, } \\
\text { 5, CEP290, } \\
\text { others }\end{array}$ \\
\hline
\end{tabular}

JSRD, Joubert syndrome and related disorders; MTS, Molar tooth sign; OMA, oculomotor apraxia; SLS, Senior-L øken syndrome; +, present; -, absent; $(+)$, sometimes present; ?, unknown or not described.

Derived from Parisi and Glass ${ }^{6}$, Gleeson et $a l^{8}$, Chance et al ${ }^{10}$, Satran et al. ${ }^{11}$

${ }^{a}$ Classic Joubert syndrome is defined as the MTS, hypotonia, developmental delay/mental retardation, +/- OMA, +/- breathing abnormalities.

${ }^{b} \mathrm{COACH}$, cerebellar vermis hypoplasia, oligophrenia, ataxia, colobomas, hepatic fibrosis.

${ }^{\circ} \mathrm{OFD}$, oral-facial-digital syndrome.

dLCA, Leber congenital amaurosis; severe congenital retinal blindness.

eSome individuals with congenital oculomotor apraxia (Cogan syndrome), some of whom have developed nephronophthisis, have been found to have NPHP1 deletions and/or NPHP1 point mutations and may actually have a mild form of JS, although the molar tooth sign was not reported. ${ }^{36}$

fEpisodic tachypnea and/or apnea.

${ }^{9}$ One entity termed Malta syndrome is characterized by hydrocephalus, cortical renal cysts, coloboma, severe retinal impairment, and occipital encephalocele; 8,14 however, it is unclear that this represents a distinct JSRD as all of these features have been described in individuals with DekabanArima syndrome. In several families with more than one affected child, encephalocele has been a discordant finding among the siblings, suggesting it is premature to define a subgroup based primarily on this CNS anomaly (M Parisi, personal observations).

holymicrogyria has been described in two subjects with AHI1 mutations and in a few individuals with no identified genetic cause. It has been hypothesized that IS with polymicrogyria represents a distinct JSRD, ${ }^{8}$ but this designation awaits further clarification.

'May include agenesis of the corpus callosum, Dandy-Walker malformation, heterotopias, or cortical dysplasia.

Joubert syndrome with retinal dystrophy and renal symptoms is sometimes designated type B.

${ }^{k}$ The distinction between cystic dysplastic kidneys and nephronophthisis at a histological level may not be clear. ${ }^{27}$

'Polydactyly can be postaxial, preaxial, or central (with a Y-shaped metacarpal).

Occipital encephaloceles or meningoceles have been observed, ${ }^{2,4,8}$ as well as agenesis of the corpus callosum. ${ }^{14}$ Polymicrogyria and/or cortical dysplasia has been described in a few subjects with $A H I 1$ mutations (see below under Molecular and genetic basis of disease), ${ }^{8,15}$ but does not appear to be a common finding in JSRD, nor among a wider cohort of subjects with AHI1 mutations. Cerebellar heterotopias have also been noted. ${ }^{2}$

Although cognitive disability can range from normal IQ to severe impairment, it is usually moderate in severity. In one series, the average age of independent sitting was 19 months and the average age of walking was 4 years for those who developed these skills; ${ }^{5}$ another series reported three outcomes: early mortality, those with developmental quotients $<30$, and those with developmental quotients $60-85 .^{3}$ Ataxia and speech dyspraxia are typical and likely due to the cerebellar malformation. Some children with JS have seizures. A few children with autism have been reported ${ }^{16,17}$ although other surveys suggest that classic autism is not typical in JS. ${ }^{18}$ Behavioral disturbances, when present, are most likely to include impulsivity and temper tantrums. ${ }^{19}$

\section{Skeletal}

Skeletal defects in JS can include polydactyly, observed in $16 \%$ of subjects in one survey. ${ }^{2}$ Postaxial polydactyly is most common, although preaxial polydactyly of the toes has been observed. Mesaxial polydactyly (occurring between the middle digits and sometimes associated with Y-shaped metacarpals) has been described in rare cases where other signs of oral-facial-digital type VI syndrome (OFD VI, sometimes called Varadi-Papp syndrome) are present. ${ }^{8}$ Children with abnormal tone may also develop orthopedic complications such as scoliosis. 


\section{Ophthalmologic}

Ocular manifestations in JS are highly variable. Disturbances of ocular motility such as OMA are very common and were documented in all children evaluated with comprehensive ophthalmologic testing in one survey. ${ }^{20}$ OMA typically manifests with dysconjugate eye movements and head thrusting to compensate for the inability to initiate saccades. ${ }^{19}$ Nystagmus (horizontal, torsional, and/or rotatory) is often present at birth and may improve with age. Strabismus, amblyopia, and ptosis may require medical or surgical intervention. Third nerve palsy has also been observed. ${ }^{19}$ Retinal disease consisting of a pigmentary retinopathy has been documented in some children with $\mathrm{JS},{ }^{2,3}$ and some infants manifest a severe form of congenital blindness with markedly flattened electroretinogram (ERG) traces, analogous to what is termed Leber congenital amaurosis. ${ }^{20,21}$ Ocular colobomas are present in some children at birth and usually affect the choroid and retina; ${ }^{2}$ these are a common feature of the JSRD known as COACH syndrome. ${ }^{8,22}$

\section{Renal}

Renal disease has been reported in up to $30 \%$ of subjects with JS. ${ }^{2}$ There are two broad categories of renal impairment: cystic dysplasia and juvenile nephronophthisis. Cystic dysplasia appears as multiple cysts of various sizes in immature kidneys with fetal lobulations on ultrasound and may be present at birth. ${ }^{2,11,23,24}$ This finding is characteristic of the JSRD known as Dekaban-Arima syndrome (Table 1). In contrast, juvenile nephronophthisis is characterized by microscopic cysts affecting the renal tubules and usually presents with urine concentrating defects in the first or second decade of life manifested by polydipsia, polyuria, anemia, and growth failure, with progression to end-stage renal disease by $\sim 13$ years of age. $^{25,26}$ Small, scarred, and echogenic kidneys on ultrasound are only visible late in the course of the disease. Although these two renal lesions have been considered distinct, one report reviewed the renal pathology that had been described as cystic dysplasia in subjects with DekabanArima syndrome and found it to be indistinguishable from nephronophthisis. ${ }^{27}$ Thus, it is possible that the renal manifestations in JSRD represent a continuum. The renal cystic disease recently described in some subjects with JS due to AHI1 mutations suggests that the onset of a nephronophthisis-like disorder may be delayed to as late as the third decade. ${ }^{28} \mathrm{~A}$ general association between retinal and renal involvement has been observed, although this is not absolute. ${ }^{2,23,29}$

\section{Hepatic}

Approximately $6 \%$ of our cohort has developed hepatic fibrosis, often presenting with elevated liver enzymes or hepato(spleno)megaly (M Parisi, personal observations). In some cases, hepatic fibrosis has slowly progressed to end- stage and required transplantation. An association with ocular colobomas has been described under the acronym COACH syndrome (see Table 1). ${ }^{22,30}$

\section{Other}

Endocrine abnormalities have been documented in a minority of subjects with JS ranging from micropenis to isolated growth hormone deficiency or panhypopituitarism (M Parisi, personal observations). Oral frenulae and tongue hamartomas have been described in the OFD VI group of disorders ${ }^{8}$ (see Table 1). A 'typical' dysmorphic facial appearance, including a broad forehead, arched eyebrows, ocular hypertelorism, and open, tent-shaped mouth, has been observed. ${ }^{5}$ Efforts to quantify facial features distinctive for JS have not been successful.

\section{Diagnostic approaches Initial evaluation}

- A high-quality MRI scan, including axial, coronal, and sagittal views (ideally with $3 \mathrm{~mm}$ axial planes through the midbrain and pons), is the most essential study in the diagnostic evaluation for a child suspected of having JS (Figure 1). CT scans in some situations have demonstrated the MTS, although this imaging modality is less sensitive and reliable. In addition to the MTS, images should be reviewed for other findings, such as polymicrogyria, callosal abnormalities, heterotopias, and evidence of ventriculomegaly or a posterior fossa fluid collection suggestive of Dandy-Walker malformation. ${ }^{1,7,31}$

- Medical genetics evaluation to obtain family history and to perform a physical examination to look specifically for polydactyly, micro/macrocephaly, facial dysmorphism, clefts, lingual nodules, abnormal genitalia, and other anomalies that may distinguish JSRD from other conditions in the differential, such as Meckel-Gruber syndrome, Bardet-Biedl syndrome, and congenital disorders of glycosylation.

- Neurological evaluation to assess cerebellar function. An EEG is indicated if clinical seizures are suspected.

- Evaluation of oromotor function by a trained occupational or speech therapist. In some children with severe hypotonia and/or dyscoordinated feeding behaviors, use of nasogastric feeding tubes or gastrostomy placement may be necessary to prevent aspiration and provide adequate caloric intake.

- Comprehensive developmental assessment to evaluate cognition, communication, and motor skills for the purpose of organizing developmental interventions.

- Sleep history with attention to apnea/hyperpnea. Polysomnogram in all children diagnosed under the age of 12 months to differentiate breathing pauses due to obstructive events (such as a hypotonic airway or 
enlarged tonsils) from centrally mediated brainstem dysregulation more typical of $\mathrm{JS}^{5}$ After the age of 1 year, this test may be useful if the child has symptoms of sleep apnea. Rarely, mechanical ventilation and tracheostomy may be considered in children with severe respiratory dysfunction.

- A baseline ophthalmologic exam is recommended to evaluate for eye malformations, such as coloboma, signs of a retinopathy or blindness, and/or eye movement abnormalities such as nystagmus or OMA. Specific ocular motility examination in a specialized laboratory (with electrooculogram in older children) may be helpful to define these eye movement disturbances. A visual evoked potential study may be a useful test that does not require sedation and can be performed as early as 6 months of age. Initial ERG studies to evaluate retinal function are recommended between the ages of 8 months and 3 years, when the sedation required is minimal. Repeat studies may be necessary if there are concerns of retinal dystrophy.

- Abdominal ultrasound scan with attention to the kidneys and liver. As the majority of infants will not manifest renal cystic disease or liver fibrosis at this young age, interval reevaluation is advised.

- Renal evaluation: blood urea nitrogen (BUN), creatinine, complete blood count (CBC) for anemia, and firstmorning void urinalysis with specific gravity to evaluate for concentrating ability (particularly in older children). A baseline blood pressure should also be obtained.

- Liver function tests (LFTs) to include transaminases, albumin, bilirubin, prothrombin time.

- Genetic testing may include a G-banded chromosome study, as part of the standard evaluation for an individual with mental retardation and other birth defects. However, no consistent chromosomal aberrations have been identified in subjects with JS. The evaluation of a hypotonic infant often includes DNA testing for Prader-Willi syndrome and other causes of hypotonia, but is expected to be normal in subjects with JSRD.
- Referral to the Joubert Syndrome Foundation and Related Cerebellar Disorders (http://www.joubertsyndrome. org/) and other support organizations for families of children with disabilities.

- The following sections describe the details of specific molecular genetic testing.

\section{Molecular and genetic basis of the disease Three causative genes}

Mutations in the NPHP1, AHI1, and CEP290 genes have been identified in some subjects with JSRD (Table 2).

\section{NPHP1}

The 30-exon NPHP1 (Nephronophthisis 1) gene and a portion of another gene of unknown function reside within a homozygous deletion of $\sim 290 \mathrm{~kb}$ flanked by large inverted repeat elements on chromosome 2q13 that has been identified in the renal disorder, juvenile nephronophthisis. ${ }^{26,32,33}$ Compound heterozygosity for the deletion combined with a point mutation in the NPHP1 gene has been identified in some subjects with nephronophthisis. ${ }^{34}$ The common NPHP1 deletion has also been identified in a few patients with $\mathrm{JS},{ }^{35,37,38}$ as well as some individuals with congenital OMA known as Cogan syndrome. ${ }^{36}$ The deletion appears to be identical in the different disorders. The NPHP1 mutation detection rate for the purely renal disorder is approximately $30 \%,{ }^{34}$ in contrast to a rate of approximately $1-2 \%$ in patients with JS based on several case series. ${ }^{28,35,37}$ However, the likelihood of detecting a causative NPHP1 deletion may be increased in subjects with JS who also have juvenile nephronophthisis. Two subjects with the homozygous NPHP1 deletion had retinal dystrophy in addition to nephronophthisis, comprising Senior-Løken syndrome. ${ }^{37,38}$ Thus far, the MTS in these individuals appears to be less striking with less extensive cerebellar vermis hypoplasia and elongated, but not thickened, superior cerebellar peduncles (Figure 2); the significance of this finding is not clear, as few JS subjects

Table 2 Genetic loci and genes for Joubert syndrome and related disorders

\begin{tabular}{|c|c|c|c|c|}
\hline Locus & Gene/mutation & $\begin{array}{l}\text { Mutation detection } \\
\text { rate }\end{array}$ & Clinical associations & References \\
\hline 2q13 NPHP1/JBTS4 & NPHP1 Homozygous deletion & $2 / 117=2 \%{ }^{28, a}$ & $\begin{array}{l}\text { Juvenile nephronophthisis, } \\
\text { OMA, +/-RD, mild MTS }\end{array}$ & $28,35,37,38$ \\
\hline $6 q 23.3$ JBTS3 & $\begin{array}{l}\text { AHI1 Many different sequence } \\
\text { variants }\end{array}$ & $13 / 117=11 \%^{28}$ & $\begin{array}{l}\mathrm{RD},+/-\mathrm{PMG} \text {, later renal } \\
\text { impairment? }\end{array}$ & $15,28,39-43$ \\
\hline $12 q 21.32$ JBTS5 & $\begin{array}{l}\text { CEP290 Mostly nonsense/ } \\
\text { frameshift mutations }\end{array}$ & $7 / 96=7 \%{ }^{44}$ & $\begin{array}{l}\text { RD, LCA, nephronophthisis; } \\
+/ \text { - coloboma, encephalocele }\end{array}$ & 44,45 \\
\hline $9 q 34.3$ JBTS1 & Unk & Unk & $\mathrm{RD} ?$ & 51 \\
\hline 11p12-11q13.3 JBTS2/CORS2 & Unk & Unk & RD?, nephronophthisis & 9,53 \\
\hline
\end{tabular}

LCA, Leber congenital amaurosis; OMA, oculomotor apraxia; PMG, polymicrogyria; RD, retinal dystrophy; Unk, unknown.

${ }^{a}$ This calculation is comparable with results obtained by Castori $e t \mathrm{al}^{3}{ }^{3}$ who found a homozygous deletion of NPHP1 in one out of 40 probands with JS. 
with NPHP1 deletions have been identified. As the typical onset of renal symptoms occurs in the second decade of life, monitoring for renal disease is required throughout childhood and early adulthood. It remains to be elucidated how a single molecular lesion (the NPHP1 deletion) can lead to the diverse phenotypes of nephronophthisis alone, OMA alone, or JSRD.

\section{AHI1}

The AHI1 (Abelson Helper-Integration site 1) gene has 28 exons (26 coding), encodes a protein called Jouberin, and was found to harbor JS-causing mutations based on mapping studies in several inbred families. ${ }^{15,39,40}$ A variety of nonsense, missense, splice-site, and insertion mutations have been identified in JS subjects ${ }^{15,28,39,41-43}$ (Figure 3). Missense mutations cluster within the WD40 domains implicated in protein-protein interactions. No single founder mutation has been identified in families of shared ethnicity. In a cohort of 117 JS subjects screened for AHI1 mutations by a combination of haplotype analysis and gene sequencing, $11 \%$ had mutations. ${ }^{28}$ Polymicrogyria of the cerebral cortex has been observed in two individuals with AHI1 mutations. ${ }^{15}$ Retinal dystrophy was reported in 11 out of 12 families with $A H I 1$ mutations; ${ }^{28}$ however, no subjects exhibited the variable JSRD features of encephalocele, polydactyly, coloboma, or liver fibrosis. Renal disease consistent with nephronophthisis has been observed in several older subjects with JS and AHI1 mutations. ${ }^{28,42}$ Thus, individuals with $A H I 1$ mutations are at risk to develop both retinal dystrophy and progressive kidney disease.

\section{CEP290}

The 55-exon CEP290 gene (Centrosomal Protein, $290 \mathrm{kDa}$ ) was identified as causative in seven of 96 individuals with JSRD in one series (7\%). ${ }^{44}$ The affected individuals in the majority of families have had retinal dystrophy or congenital blindness, and renal disease consistent with nephronophthisis or renal cortical cysts has also been present. ${ }^{44,45}$ Thus, the form of JSRD in many families with CEP290 mutations overlaps with Senior-Løken syndrome or Joubert-LCA like. Other findings in some affected individuals include ocular colobomas and encephaloceles. ${ }^{44}$ Almost all of the JSRD-associated CEP290 mutations have included nonsense, splice-site, or insertions/ deletions resulting in frameshift mutations. ${ }^{43,44}$ Mutations in CEP290 have also been identified in $~ 21 \%$ of patients with Leber congenital amaurosis without the MTS or

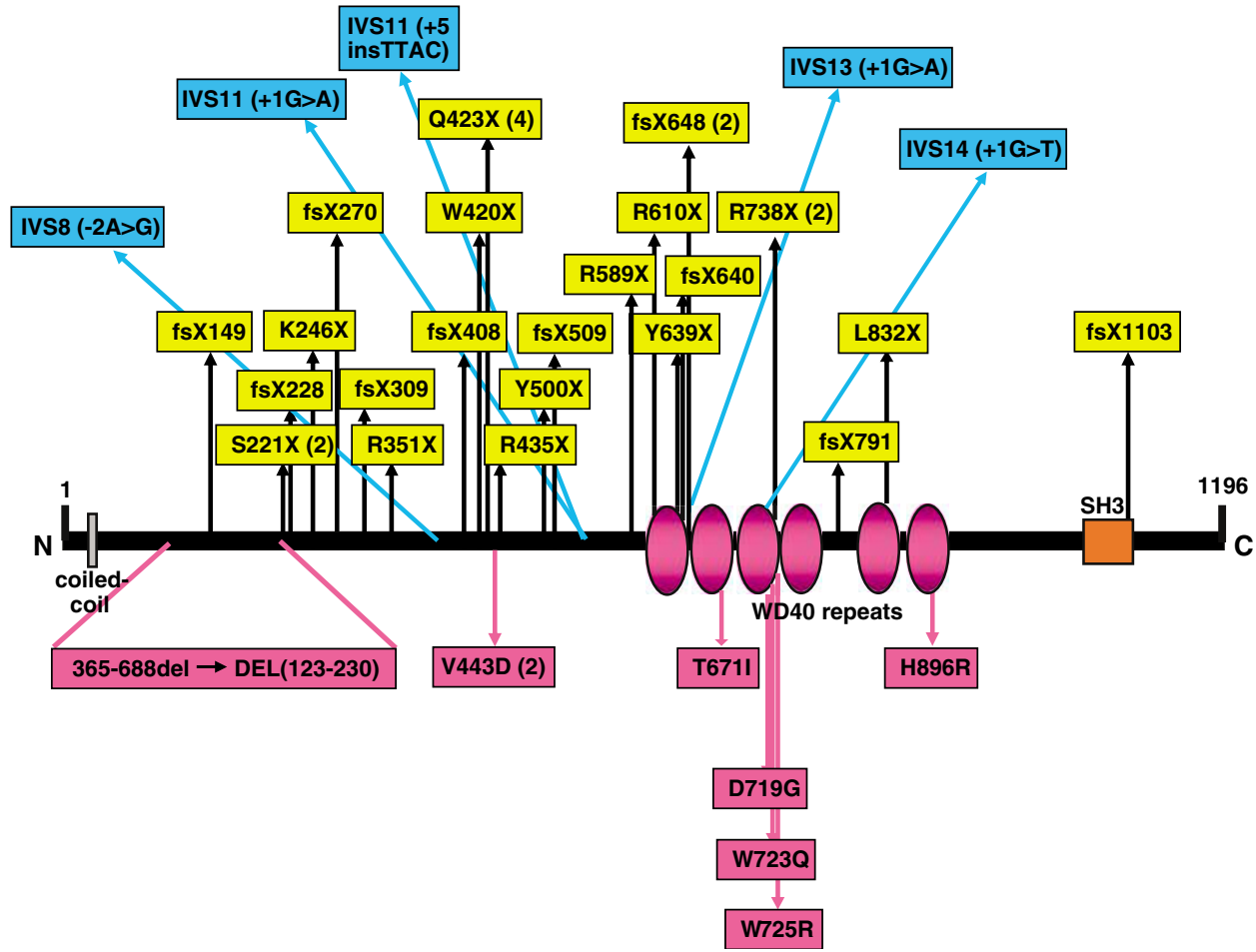

Figure 3 AHI1 protein with identified mutations. Mutations resulting in protein termination are indicated in yellow boxes above the protein. Splice-site mutations are indicated by blue boxes above the protein, starting with 'IVS' to designate the intronic change. Missense mutations and one in-frame deletion are indicated as pink boxes below the protein. Protein motifs include an amino-terminal coiled-coil domain, six WD40 domains, and a carboxy-terminal Src-homology $3(\mathrm{SH} 3)$ domain. Numbers in parentheses indicate number of families in which the mutation has been identified. From Dixon-Salazar et $a l^{15}$, Parisi et $a l^{28}$, Ferland et $a l^{39}$, Romano et $a l^{41}$, Utsch et $a l^{42}$, Valente et $a l^{43}$ 
cognitive impairment; in these individuals, a common point mutation creates an aberrant splice product but some residual production of wild-type protein is hypothesized to account for the milder phenotype than the complete lossof-function mutations found in JSRD patients. ${ }^{46}$

\section{Potential role of these genes in the primary cilium/centrosome}

NPHP1 and other nephrocystin gene products associated with juvenile nephronophthisis are known to localize to the cell-cell junctions and the primary cilium and basal body apparatus of renal epithelial cells. ${ }^{26,47}$ These nephrocystin gene products form multiprotein complexes with microtubules, adherens proteins, and other cytoskeletal components, and may play a role as mechanosensors in cellular signaling processes or in intraflagellar transport. ${ }^{26}$ Although a precise role for these proteins has not yet been elucidated in the cerebellum, it is known that most neurons and glia in the mammalian brain contain a primary cilium, an organelle that is present on almost all cells. $^{48,49}$ A role for the AHI1 protein in ciliary function has not been identified. However, CEP290 localizes to the centrosome at the base of the cilium and is present in the cerebellum during mouse embryogenesis; expression defects in zebrafish result in aberrant retinal, cerebellar, and renal development. ${ }^{44,45}$ The centrosome is known to be important in chromosome segregation and may play a role in cell-cycle regulation. The functions of this complex organellar structure are many and varied, and mutations in proteins that form the centrosome/basal body apparatus and primary cilium are emerging as important causes of diseases such as Bardet-Biedl syndrome, OFD syndrome 1, Meckel-Gruber syndrome polycystic kidney disease, and lissencephaly, ${ }^{47,50}$ some of which have clinical overlap with JSRD.

\section{Other loci}

The NPHP1 and AHI1 genes combined accounted for $<15 \%$ of causative mutations in a cohort of 117 subjects, strongly suggesting that other genes for JS remain to be identified. ${ }^{28}$ In another study, the CEP290 gene was causative in seven out of 96 individuals with JSRD (7\%). ${ }^{44}$ Linkage analysis has implicated two additional loci in JSRD (Table 2). Two consanguineous Arab families from Oman showed linkage to $9 q 34,{ }^{51}$ identifying the JBTS1 locus. Several of these affected individuals developed retinal dystrophy, but renal disease has not been described. ${ }^{14,52}$ An extended consanguineous Italian family ${ }^{9}$ and several Arab families ${ }^{53}$ showed linkage to a locus on the pericentromeric region of chromosome 11, termed the CORS2 (JBTS2) locus. Many of these subjects developed renal disease, and some had retinal dystrophy. ${ }^{14}$ The genes at these loci are not known. The identification of consanguineous families that do not map to any of these five loci suggests the presence of additional causative genes. ${ }^{14,54,55}$

\section{Management \\ Clinical work-up (see 'Diagnostic approaches' section for details of Initial Evaluation) \\ Surveillance and management Given the heterogeneity in JSRD and the relatively high frequency of associated medical conditions, ongoing monitoring is essential. As the genetic causes of these disorders are identified, genotype-phenotype correlations may make it possible to refine these recommendations. The medical advisory board of the JS parent advocacy organization has deve- loped recommendations for surveillance and management in this group of disorders (http://www.joubertsyndrome. com/). The management for each individual with JS must be individualized based on his/her specific medical needs and issues.}

\section{Regular evaluations}

- Annual pediatric and neurological evaluation to monitor systemic or motor abnormalities.

- Annual monitoring of growth and sexual maturation with endocrinologic evaluation if indicated.

- Patients with progressive kidney failure may present with reduced urine concentrating ability or anemia. Therefore, CBC, first-morning void urinalysis, BUN, creatinine, abdominal ultrasound scan, as well as blood pressure measurement, are recommended annually until at least age 20 years.

- Ophthalmologic abnormalities may be progressive. Therefore, annual retinal examination starting at the time of diagnosis is recommended with follow-up ERG if indicated by the ophthalmologist.

- Periodic developmental assessments to help design therapy interventions and school programs and evaluate for assistive equipment needs.

- Neuropsychological evaluation if indicated for behavioral issues.

- Annual liver evaluation by ultrasound (as for kidneys) and LFTs (transaminases, albumin, bilirubin, prothrombin time) initiating at the time of diagnosis.

- Patients may have problems with swallowing due to large tongues, and may need ongoing evaluation for oromotor function and polysomnography for symptoms of obstructive apnea.

Genetic testing strategy As it is possible that an autosomal aneusomy condition could lead to features similar to those in JS (such as hypotonia and developmental delay), karyotype analysis and specialized genetic testing may be pursued in some cases (see Diagnostic approaches and Figure 1). However, an MRI should confirm the MTS, and 
chromosomal anomalies have not been a feature in JS. Testing for mutations in the three genes that have been identified as causative for JSRD (Table 2) should be considered, depending on clinical features and availability. Clinical molecular testing for the homozygous deletion of the NPHP1 gene associated with juvenile nephronophthisis, $^{35,56}$ identified in only $1-2 \%$ of subjects with JS, is available by FISH or microsatellite marker analysis ${ }^{28}$ (see http://www.genetests.org for a list of testing laboratories). Molecular testing for mutations in the AHI 1 gene and the CEP290 gene is also available clinically; each of these genes is estimated to account for $\sim 10 \%$ of cases of JSRD. Mutations in both AHI1 and CEP290 are more likely to be associated with retinal disease, with CEP290 implicated in some cases with congenital blindness or Leber amaurosis. ${ }^{44,46}$ In rare families with a large number of affected children and/or known consanguinity, linkage analysis may be feasible on a research basis.

Genetic counselling JS (and JSRD) are inherited in an autosomal recessive manner in all reported families where inheritance pattern can be determined. The penetrance for this disorder appears to be $100 \%$, although data are limited to known linkage and mutational surveys. A couple who has had one affected child has a $25 \%$ chance of giving birth to another child with JS in each subsequent pregnancy. For an unaffected sibling of an individual with JS, the probability of being a carrier is $2 / 3$. Based on an estimated prevalence of $1: 100000$, the carrier frequency in the general population is calculated as $\sim 1: 160$. This results in a $(1 / 160)(1 / 2)(2 / 3)(1 / 2)=1 / 960$ chance for an unaffected sibling to have a child with JS. This would be higher in some ethnic groups or in the presence of consanguinity. There are no reports of an individual with JS reproducing in the medical literature; however, the probability that a person with JS would have an affected offspring would be quite low assuming the partner was neither affected nor a carrier for JS. Although a few individuals with JS are mildly affected, in general, JS is a moderately severe disorder with major impacts on health and development. The variable severity of the disorder in different families and even between individuals within families makes counseling regarding the prognosis challenging. ${ }^{57}$

Prenatal diagnosis For the rare families in which diseasecausing mutations have been identified, prenatal diagnosis by DNA testing is possible. For all other at-risk pregnancies, prenatal imaging is the best and most practical diagnostic option. In the presence of a family history, prenatal diagnosis of JS has been accomplished as early as the first trimester on the basis of extracranial anomalies, such as polydactyly or renal cysts and major structural CNS malformations such as encephalocele. ${ }^{58}$ Early diagnosis is more challenging when extracranial abnormalities are not present, because cerebellar vermis hypoplasia cannot be reliably diagnosed until 18-20 weeks gestation, ${ }^{59}$ and the MTS has not been observed before 27 weeks gestation. ${ }^{60}$ In the absence of a family history, one example of prenatal diagnosis has been published; at 32 weeks gestation, Aslan et $a l^{61}$ observed vermian agenesis, bilateral ventriculomegaly, postaxial polydactyly, and episodes of tachypnea, strongly suggesting a diagnosis of JS. An imaging protocol for prenatal diagnosis has been proposed; however, sensitivity and specificity of prenatal imaging findings for JSRD are not known. ${ }^{62}$

Treatment and care No specific treatments for JS exist. The mainstay of care is supportive with standard interventions for individuals with developmental disabilities, such as special education programs, physical, occupational, and speech therapy, adaptive equipment as needed, and seizure management if necessary. See recommendations above under Surveillance and Management. Potential medical treatments for some associated conditions are emerging:

- Nephronophthisis: Dialysis and renal transplantation. There may be medications available in the future to treat renal insufficiency; for instance, vasopression V2 antagonists have been shown to slow the progression of renal tubular disease in rat models of polycystic kidney disease. $^{63}$

- Liver fibrosis: Specific surgical interventions for esophageal varices or portal hypertension. Some individuals with $\mathrm{COACH}$ syndrome have undergone successful liver transplantation.

- Retinal dystrophy: Gene therapy in animal models of Leber congenital amaurosis and retinitis pigmentosa may lead to future therapies to slow the development and progression of retinal disease in humans. ${ }^{64}$

\section{Conclusion}

The major challenges in the field of JS research remain identification of the causative genes and translation of research findings into practical clinical guidelines and treatments for patients and families. Great strides have been made in this field over the past 2 years as the first causative genes have been discovered. However, conservative estimates suggest that the genetic etiology of $\sim 75 \%$ of JSRD remains to be discovered. Creative solutions will be necessary to identify the genetic causes for a condition with such a large degree of clinical and genetic heterogeneity. Fortunately, some genotype-phenotype correlations are emerging, and it is hoped that as more genes responsible for JSRD are identified, this information will allow refinement of management recommendations, so that lifelong screening for potential complications is not necessary for all patients. 
It is intriguing that this disorder is associated with both congenital anomalies (ie, brain malformations, colobomas, polydactyly), and future risks to develop progressive retinal dystrophy, renal failure, and hepatic failure. In this regard, JS is remarkably similar to Bardet-Biedl syndrome, with at least 11 different loci/genes identified, whose gene products all play a role in the basal body/ciliary apparatus. ${ }^{65}$ Discovery of genes for JS may provide further information about the function of the centrosome, basal body, and primary cilium and may also lead to valuable insights into mechanisms of cerebellar, renal, and ocular development.

From a clinical perspective, relatively little is known about the natural history of JS, perhaps because the condition is uncommon and was first reported relatively recently, in 1969. Additional clinical studies to describe the evolution of oculomotor, retinal, renal, and hepatic complications are urgently needed. Detailed characterization of the behavioral and neuropsychological phenotypes of genetic disorders such as JS will be crucial to developing effective interventions. It is hoped that an understanding of the genetic basis of JS will also facilitate development of targeted therapeutics for the associated medical complications.

\section{Note added in proof}

Recently, mutations in the TMEM67(MKS3) gene at 8q24, found to be causative in Meckel-Gruber syndrome, have been identified in three individuals with JS. While all of the affected individuals manifest cerebellar vermis hypoplasia and/or the MTS, two of them lack the classic features of Meckel-Gruber syndrome (encephalocele, renal cysts, Polydactyly, and hepatic fibrosis). ${ }^{66}$ Clinical testing for mutations in MKS3 is available.

\section{Acknowledgements}

We thank the patients and their families and the Joubert Syndrome Foundation \& Related Cerebellar Disorders for participating in research studies. We acknowledge the helpful discussions and contributions of Jonathan $R$ Adkins, BS, Craig L. Bennett, PhD, William B Dobyns, MD, Melissa L Eckert, BS, Nicholas T Gorden, BS, Dana Knutzen, MS, Dennis WW Shaw, MD, and many clinical and research collaborators. This work was supported by National Institutes of Health grants P30-HD02274, K23-NS45832 (MAP), and K24HD46712 (IAG); the March of Dimes Endowment for Healthier Babies at Children's Hospital in Seattle; and the Center for Neurogenetics and Neurotherapeutics, University of Washington.

\footnotetext{
References

1 Maria BL, Quisling RG, Rosainz LC et al: Molar tooth sign in Joubert syndrome: clinical, radiologic, and pathologic significance. J Child Neurol 1999; 14: 368-376.

2 Saraiva JM, Baraitser M: Joubert syndrome: a review. Am J Med Genet 1992; 43: 726-731.

3 Steinlin M, Schmid M, Landau K, Boltshauser E: Follow-up in children with Joubert syndrome. Neuropediatr 1997; 28: 204-211.
}

4 Joubert M, Eisenring JJ, Robb JP, Andermann F: Familial agenesis of the cerebellar vermis. A syndrome of episodic hyperpnea, abnormal eye movements, ataxia, and retardation. Neurology 1969; 19: 813-825.

5 Maria BL, Boltshauser E, Palmer SC, Tran TX: Clinical features and revised diagnostic criteria in Joubert syndrome. J Child Neurol 1999; 14: 583-590; discussion 590-591.

6 Parisi MA, Glass IA: Joubert syndrome; In: GeneReviews at GeneTests-GeneClinics: Medical Genetics Information Resource [database online]. Copyright, University of Washington, Seattle. 1997-2006. Available at http://www.geneclinics.org or http:// www.genetests.org, 2006.

7 Maria BL, Hoang KB, Tusa RJ et al: 'Joubert syndrome' revisited: key ocular motor signs with magnetic resonance imaging correlation. J Child Neurol 1997; 12: 423-430.

8 Gleeson JG, Keeler LC, Parisi MA et al: Molar tooth sign of the midbrain-hindbrain junction: occurrence in multiple distinct syndromes. Am J Med Genet 2004; 125A: 125-134; discussion 117

9 Valente EM, Salpietro DC, Brancati F et al: Description, nomenclature, and mapping of a novel cerebello-renal syndrome with the molar tooth malformation. Am J Hum Genet 2003; 73: 663-670.

10 Chance PF, Cavalier L, Satran D, Pellegrino JE, Koenig M, Dobyns WB: Clinical nosologic and genetic aspects of Joubert and related syndromes. J Child Neurol 1999; 14: 660-666; discussion 669-672.

11 Satran D, Pierpont ME, Dobyns WB: Cerebello-oculo-renal syndromes including Arima, Senior-Loken and $\mathrm{COACH}$ syndromes: more than just variants of Joubert syndrome. Am J Med Genet 1999; 86: 459-469.

12 Maria BL, Bozorgmanesh A, Kimmel KN, Theriaque D, Quisling RG: Quantitative assessment of brainstem development in Joubert syndrome and Dandy-Walker syndrome. J Child Neurol 2001; 16: 751-758.

13 Genel F, Atlihan F, Ozdemir D, Targan S: Development of hydrocephalus in a patient with Joubert syndrome. I Postgrad Med 2004; 50: 153.

14 Valente EM, Marsh SE, Castori M et al: Distinguishing the four genetic causes of jouberts syndrome-related disorders. Ann Neurol 2005; 57: 513-519.

15 Dixon-Salazar T, Silhavy JL, Marsh SE et al: Mutations in the AHI1 gene, encoding Jouberin, cause Joubert syndrome with cortical polymicrogyria. Am J Hum Genet 2004; 75: 979-987.

16 Holroyd S, Reiss AL, Bryan RN: Autistic features in Joubert syndrome: a genetic disorder with agenesis of the cerebellar vermis. Biol Psychiatry 1991; 29: 287-294.

17 Ozonoff S, Williams BJ, Gale S, Miller JN: Autism and autistic behavior in Joubert syndrome. J Child Neurol 1999; 14: 636-641.

18 Takahashi TN, Farmer JE, Deidrick KK, Hsu BS, Miles JH, Maria BL: Joubert syndrome is not a cause of classical autism. Am J Med Genet A 2005; 132: 347-351.

19 Hodgkins PR, Harris CM, Shawkat FS et al: Joubert syndrome: long-term follow-up. Dev Med Child Neurol 2004; 46: 694-699.

20 Tusa RJ, Hove MT: Ocular and oculomotor signs in Joubert syndrome. J Child Neurol 1999; 14: 621-627.

21 Ivarsson SA, Bjerre I, Brun A, Ljungberg O, Maly E, Taylor I: Joubert syndrome associated with Leber amaurosis and multicystic kidneys. Am J Med Genet 1993; 45: 542-547.

22 Verloes A, Lambotte C: Further delineation of a syndrome of cerebellar vermis hypo/aplasia, oligophrenia, congenital ataxia, coloboma, and hepatic fibrosis. Am J Med Genet 1989; 32: $227-232$.

23 Steinlin M, Blaser S, Boltshauser E: Cerebellar involvement in metabolic disorders: a pattern-recognition approach. Neuroradiology 1998; 40: 347-354.

24 Dekaban AS: Hereditary syndrome of congenital retinal blindness (Leber), polycystic kidneys and maldevelopment of the brain. Am J Ophthalmol 1969; 68: 1029-1037. 
25 Hildebrandt F, Nothwang HG, Vossmerbaumer $\mathrm{U}$ et al: Lack of large, homozygous deletions of the nephronophthisis 1 region in Joubert syndrome type B. Pediatr Nephrol 1998; 12: $16-19$.

26 Saunier S, Salomon R, Antignac C: Nephronophthisis. Curr Opin Genet Dev 2005; 15: 324-331.

27 Kumada S, Hayashi M, Arima K et al: Renal disease in Arima syndrome is nephronophthisis as in other Joubert-related Cerebello-oculo-renal syndromes. Am J Med Genet A 2004; 131: $71-76$.

28 Parisi MA, Doherty D, Eckert ML et al: AHI1 mutations cause both retinal dystrophy and renal cystic disease in Joubert syndrome. J Med Genet 2006; 43: 334-339.

29 King MD, Dudgeon J, Stephenson JB: Joubert's syndrome with retinal dysplasia: neonatal tachypnoea as the clue to a genetic brain-eye malformation. Arch Dis Child 1984; 59: 709-718.

30 Gentile M, Di Carlo A, Susca F et al: COACH syndrome: report of two brothers with congenital hepatic fibrosis, cerebellar vermis hypoplasia, oligophrenia, ataxia, and mental retardation. Am J Med Genet 1996; 64: 514-520.

31 Quisling RG, Barkovich AJ, Maria BL: Magnetic resonance imaging features and classification of central nervous system malformations in Joubert syndrome. I Child Neurol 1999; 14: 628-635; discussion 669-672.

32 Hildebrandt F, Otto E, Rensing C et al: A novel gene encoding an SH3 domain protein is mutated in nephronophthisis type 1. Nat Genet 1997; 17: 149-153.

33 Saunier S, Calado J, Benessy F et al: Characterization of the NPHP1 locus: mutational mechanism involved in deletions in familial juvenile nephronophthisis. Am J Hum Genet 2000; 66: $778-789$

34 Hoefele J, Otto E, Felten $\mathrm{H}$ et al: Clinical and histological presentation of 3 siblings with mutations in the NPHP4 gene. Am J Kidney Dis 2004; 43: 358-364.

35 Parisi MA, Bennett CL, Eckert ML et al: The NPHP1 gene deletion associated with juvenile nephronophthisis is present in a subset of individuals with Joubert syndrome. Am J Hum Genet 2004; 75: $82-91$.

36 Betz R, Rensing C, Otto E et al: Children with ocular motor apraxia type Cogan carry deletions in the gene (NPHP1) for juvenile nephronophthisis. J Pediatr 2000; 136: 828-831.

37 Castori M, Valente EM, Donati MA et al: NPHP1 gene deletion is a rare cause of Joubert syndrome related disorders. I Med Genet 2005; 42: e9.

38 Caridi G, Dagnino M, Rossi A et al: Nephronophthisis type 1 deletion syndrome with neurological symptoms: prevalence and significance of the association. Kidney Int 2006; 70: $1342-1347$.

39 Ferland RJ, Eyaid W, Collura RV et al: Abnormal cerebellar development and axonal decussation due to mutations in AHI1 in Joubert syndrome. Nat Genet 2004; 36: 1008-1013.

40 Lagier-Tourenne C, Boltshauser E, Breivik N et al: Homozygosity mapping of a third Joubert syndrome locus to 6q23. J Med Genet 2004; 41: 273-277.

41 Romano S, Boddaert N, Desguerre I et al: Molar tooth sign and superior vermian dysplasia: a radiological, clinical, and genetic study. Neuropediatrics 2006; 37: 42-45.

42 Utsch B, Sayer JA, Attanasio M et al: Identification of the first AHI1 gene mutations in nephronophthisis-associated Joubert syndrome. Pediatr Nephrol 2006; 21: 32-35.

43 Valente EM, Brancati F, Silhavy JL et al: AHI1 gene mutations cause specific forms of Joubert syndrome-related disorders. Ann Neurol 2006; 59: 527-534.

44 Sayer JA, Otto EA, O'Toole J F et al: The centrosomal protein nephrocystin- 6 is mutated in Joubert syndrome and activates transcription factor ATF4. Nat Genet 2006; 38: 674-681.
45 Valente EM, Silhavy JL, Brancati F et al: Mutations in CEP290, which encodes a centrosomal protein, cause pleiotropic forms of Joubert syndrome. Nat Genet 2006; 38: 623-625.

46 den Hollander AI, Koenekoop RK, Yzer S et al: Mutations in the CEP290 (NPHP6) gene are a frequent cause of Leber Congenital Amaurosis. Am J Hum Genet 2006; 79: 556-561.

47 Watnick T, Germino G: From cilia to cyst. Nat Genet 2003; 34: $355-356$.

48 Fuchs JL, Schwark HD: Neuronal primary cilia: a review. Cell Biol Int 2004; 28: 111-118.

49 Whitfield JF: The neuronal primary cilium - an extrasynaptic signaling device. Cell Signal 2004; 16: 763-767.

50 Badano JL, Teslovich TM, Katsanis N: The centrosome in human genetic disease. Nat Rev Genet 2005; 6: 194-205.

51 Saar K, Al-Gazali L, Sztriha L et al: Homozygosity mapping in families with Joubert syndrome identifies a locus on chromosome 9q34.3 and evidence for genetic heterogeneity. Am J Hum Genet 1999; 65: 1666-1671.

52 Sztriha L, Al-Gazali LI, Aithala GR, Nork M: Joubert's syndrome: new cases and review of clinicopathologic correlation. Pediatr Neurol 1999; 20: 274-281.

53 Keeler LC, Marsh SE, Leeflang EP et al: Linkage analysis in families with Joubert syndrome plus oculo-renal involvement identifies the CORS2 locus on chromosome 11p12-q13.3. Am J Hum Genet 2003; 73: 656-662.

54 Innes AM, Parboosingh J, Roccamatisi D et al: Joubert Syndrome (JS) and Meckel-Gruber syndrome (MGS) are the same disorder in the Hutterite Brethren [abstract 602]. Presented at the annual meeting of The American Society of Human Genetics, October 29, 2004, Toronto, Ontario, Canada. Available from http://www.ashg.org/ genetics/ashg04s/index.shtml, 2004.

55 Janecke AR, Muller T, Gassner I et al: Joubert-like syndrome unlinked to known candidate loci. J Pediatr 2004; 144: 264-269.

56 Hildebrandt F, Rensing C, Betz R et al: Establishing an algorithm for molecular genetic diagnostics in 127 families with juvenile nephronophthisis. Kidney Int 2001; 59: 434-445.

57 Raynes HR, Shanske A, Goldberg S, Burde R, Rapin I: Joubert syndrome: monozygotic twins with discordant phenotypes. J Child Neurol 1999; 14: 649-654; discussion 669-672.

58 Wang $\mathrm{P}$, Chang FM, Chang $\mathrm{CH}$, Yu CH, Jung YC, Huang CC: Prenatal diagnosis of Joubert syndrome complicated with encephalocele using two-dimensional and three-dimensional ultrasound. Ultrasound Obstet Gynecol 1999; 14: 360-362.

59 Bromley B, Nadel AS, Pauker S, Estroff JA, Benacerraf BR: Closure of the cerebellar vermis: evaluation with second trimester US Radiology 1994; 193: 761-763.

60 Fluss J, Blaser S, Chitayat D et al: Molar tooth sign in fetal brain magnetic resonance imaging leading to the prenatal diagnosis of Joubert syndrome and related disorders. J Child Neurol 2006; 21: 320-324.

61 Aslan H, Ulker V, Gulcan EM et al: Prenatal diagnosis of Joubert syndrome: a case report. Prenatal Diagn 2002; 22: 13-16.

62 Doherty D, Glass IA, Siebert JR et al: Prenatal diagnosis in pregnancies at risk for Joubert syndrome by ultrasound and MRI. Prenat Diagn 2005; 25: 442-447.

63 Wang X, Gattone II V, Harris PC, Torres VE: Effectiveness of vasopressin V2 receptor antagonists OPC-31260 and OPC-41061 on polycystic kidney disease development in the PCK rat. J Am Soc Nephrol 2005; 16: 846-851.

64 Dejneka NS, Rex TS, Bennett J: Gene therapy and animal models for retinal disease. Dev Ophthalmol 2003; 37: 188-198.

65 Beales PL: Lifting the lid on Pandora's box: the Bardet-Biedl syndrome. Curr Opin Genet Dev 2005; 15: 315-323.

66 Baala L, Romano S, Khaddour R et al: The Meckel-Gruber Syndrome Gene, MKS3, Is Mutated in Joubert Syndrome. Am J Hum Genet 2007; 80: 186-194. 